\title{
Design and Implementation of a Context-Aware Based Classroom
}

\section{Inquiring System}

\author{
Feng Liu ${ }^{1}$, Zhou Xu ${ }^{1}$, Chuanchao Zhang ${ }^{1}$, Haijun $\mathrm{Lu}^{1}$, Xiaoxia $\mathrm{Li}^{1{ }^{1, *}}$ \\ ${ }^{1}$ College of Science, Huazhong Agricultural University \\ Wuhan, 430070, China \\ *Corresponding author \\ E-mail: liufeng@mail.hzau.edu.cn, lixiaoxia@mail.hzau.edu.cn
}

\begin{abstract}
Nowadays, smart phones and tablets have been getting more and more popular. Many applications in various mobile platforms can provide location based service to users but seldom take personalized information into consideration which is usually important for some kinds of applications. In order to solve the popular problem of searching a available classroom on campus to do individual study and provide students with personalized services, in this paper, we design and implementation of a context-aware based available classroom searching system-CACS, which can not only use the user's location information but also takes personal preferences into consideration to make this type of applications more convenient and personalized. In this paper, the system model is constructed and some key issues and methods such as weigh based classroom evaluation algorithm are proposed. The prototype system shows that this system can benefit users with good experiences.
\end{abstract}

Keywords-context-aware applications; local based service; personal preferences; available classroom searching system

\section{INTRODUCTION}

The concept of context-aware was proposed by the researchers in the area of ubiquitous computing and now has been utilized in various applications. 'Context' refers to the physical and social situation in which computational devices are embedded[1]. Context-aware computing can provide services that are appropriate to the particular setting by acquiring and utilizing information about this context of a device[1]. The term 'context' has different meanings in different kinds of applications. For the applications developed in mobile environment it specifies the characteristics of the environment which information is going to be presented, and in artificial intelligence and real time systems, it is defined as the background and conditional information which can facilitate processing of the situations[2].

For network applications, content should not be delivered the same to different people. Application systems should provide the right content to a person with the specified demand at the specified place and at the specified time. In this area, researchers have done a lot of work.

Huang et al.[3] proposed a reliable context model for context-aware applications. Because the context information influenced by the environment status maybe imperfect, in order to deal with the incorrect context information, Huang proposed a context model to evaluate and predict the reliability of context information. In[4], an approach called context directory is presented to help mobile applications to achieve-awareness. To help users obtain their desired web services. Xiao et al. [5] proposed a context modeling approach which can automatically search and recommend services according to a user's context and can dynamic adapt to newly added context types such as location, time and activity. Ontology theory is used in their reach to enhance the meaning of a user's context values and it can help to identify the relations among different context values. To assist a M-commerce application, Pushpa et al. [6] developed a context-Information observation conceptual model to analyze the context data and infer context at a more abstract level. In this model, context information is classified into three categories: well-defined, qualitative and credible context information. Thyagaraju et al.[7] present an user context model for interactive context aware TV, which is applicable to both single and multiple user environments.

With the development of context-aware applications, the privacy issue is increasingly captured researcher's attention. Lee et al. [8] develop and then prioritize the measurement criteria for information privacy in context-aware computing environment. Pallapa et al.[9] proposed a context-aware approach to privacy preservation in wireless and mobile pervasive environments and specially two schemes are designed.

Nowadays, smart phones and tablets have been getting more and more popular. It has attracted a lot of attention that how can we utilize the mobile devices together with personal information to provide specified services for a user according to his context information. Although a plenty of personalized applications are developed for various mobile platforms which makes people's life more convenient, there are still a lot of work we should do. For example, many applications can provide location based service to help a person to find the nearest destination such as a restaurant, a gymnasium and etc but they do not use the information such as personal preferences information, which can be deduced by the history operation records.

On campus, it is a very difficult for students to search for an available classroom to do individual study. So, in this paper, we aim to design and implementation of a context- 
based system named CACS for students to solve this critical problem of searching available classroom and provide students more user-friendly experiences. Specially, we take the students' references information into consideration and students can get different services from this system according to their personalized information.

The remainder of this paper is organized as follows. In section II, we will describe the structure of CACS. In section III, the weight based available classroom searching algorithm will be discussed. We will discuss the prototype system of CACS in section IV, and draw the conclusion and describe our future work in section V.

\section{The STRUCTURE OF CACS}

\section{A. Background}

On campus, students always spend a lot of time in searching an appropriate classroom for individual study. Searching classroom one by one is a hard task for students especially during the period near the final examination of a term. So, it is necessary for us to develop an efficiency system to help students. Different with other applications, we want to recommend students the classrooms in a list according to their personalized information. In other words, different users will get different services because of their different personal context information.

\section{B. The structure of CACS}

Fig.1 illustrates the structure of CACS system. Multiple kinds of devices such as PCs, Smart Phones and Tablets can access to this system through $3 \mathrm{G}$ or Wi-Fi. Firstly, the system should recognize device categories and take their differences into consideration. For example, if a person access to the system through a smart phone, he could download the client software and install it in its smart phone, and he also could just access to the system by the browser. Particularly, the system support both C/S and B/S structure.

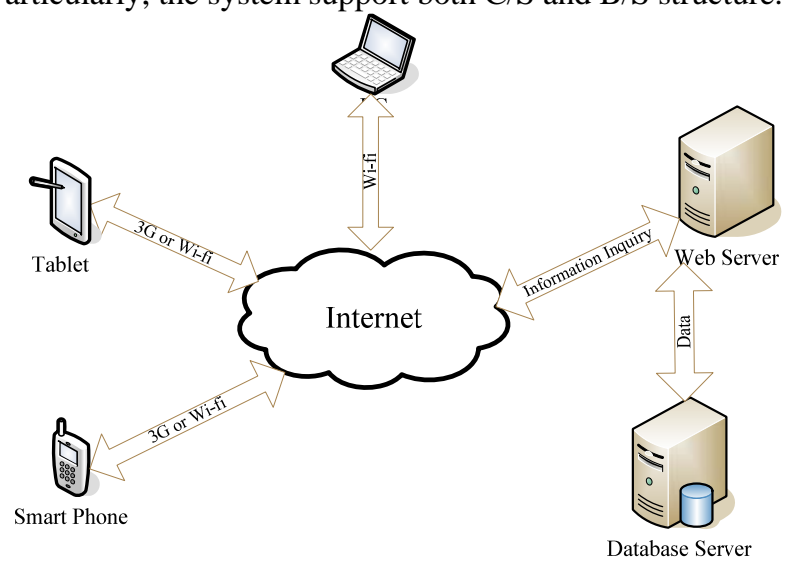

Figure 1. The structure of CACS

Specially, for the devices with a GPS module, the system should provide the location based service helping users to find the nearest available classrooms.

For a subscriber to this system, its personal information file is empty at first, but the personalized information related data will be gradually added to this file. For example, if a user likes to choose the classroom at one certain building, according to his previous behaviors, we can deduce that he will also prefer the classrooms in the same building if there is any. In this paper, we denote this kind of context information as personal profile. From what we discussed above, we can easily find that in this system, according to a user's personal profile which reflects his previous behaviors, we can present personalized available classroom searching results. By this way, a user's demand can be exactly met through this system which looks like a kind of software developed specially for a certain user.

Another problem we need to take into consideration is where personal information should to be stored. We can store them in the server or in the client. Some persons believe that personal information should be stored in the client for security and privacy consideration. But in this way, the client should have enough computational abilities to complete the task. But, it is difficult for some devices like smart phones to do this kind of work because of the limited energy and data processing ability. On the contrary, storing all the personal information in the server can also arouse users' worry about the leaking of privacy information. Consequently, in this paper, we settle a compromise to solve this issue. We encrypt personal information and store most of them in the server and some key personal information will be stored in the client, and when the user sends a searching request, this part of personal information is sent to the server together if it is necessary.

The database server showed in fig. 1 store the information about the classroom and some of the personal information of every user. When a searching request reach the web server, the web server accepts and analyzes the request, then retrieves the correlated data from the data server and computes the results. After having finished these steps, the server will provide the results to the user.

\section{Weight Based Available ClassRoom SEARChing ALGORITHM}

To develop the system of CACS, there are many key factors we should pay more attention to. In this section, we will show these key factors and propose the weight based available classroom searching algorithm

\section{A. Common factors}

In order to get the optimal results for a certain users, we should consider some key factors. In this section, we will discuss the common factors which mean these factors are related to every user. Take the available time for example, different classroom has different available time duration because there may be a course in a classroom at any time. For example, if a person chooses a classroom to do individual study, when he gets into this classroom he find that a course will be hold in this classroom twenty minutes later. So, this classroom can not be a good choice. Consequently, how long a classroom could be served as an individual studying place should be considered.

The other common factor we should take into consideration is how many students have been there. If a 
classroom is too crowded to accommodate any more it is also could not be a good choice. The system should not recommend the crowded classroom to the users.

\section{B. Personal factors}

Personal factors are related to individuals and this kind of factors should be considered individually according to students' different personal preferences. The first factor is the location. Obviously, the nearest classrooms should be recommended to users. If a person has a device with a GPS module, he can send a request together with his location information.

Another factor that we should pay more attention to is users' preferences. Some students prefer classroom in low storey while others would like classroom in high storey. Some students choose the classroom in one building, and at the same time, others would rather choose ones in the other buildings. This type of information is closely related to the students' personal preferences.

Some of the common and personal factors that we take into consideration in showed in fig.2.

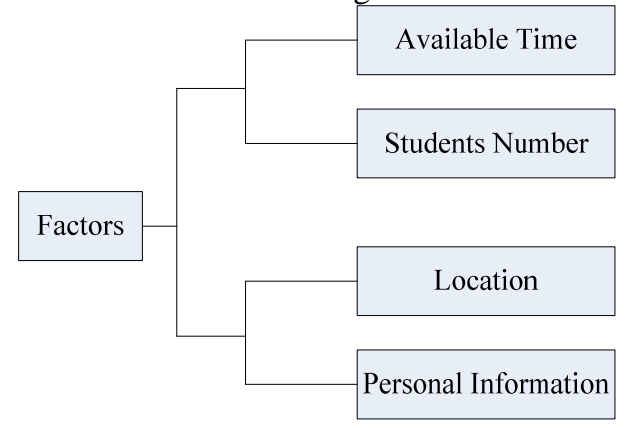

Figure 2. Factors that we should take into consideration

\section{Weight Based Available Classroom Searching Algorithm}

In section $\mathrm{A}$ and $\mathrm{B}$, we have discussed the key factors that we should take into consideration in this system. But how can we utilize these factors is another problem. In this paper, we propose a weight based available classroom searching algorithm to solve this problem.

Fig.3 illustrates the method of weight based available classroom searching method.

First, a user sends a classroom searching request to the system.

Second, the system receives his request and retrieves the personalized information and the state of every classroom from the database server. At first, we should give an experienced weight value for each factor as shown in fig. 3 and calculate the marks for each candidate classrooms through (1). In (1) $w_{i}$ represents the weight value of each factor and $m_{i}$ means the marks of each factor. The value of $m_{i}$ should be normalized.

$$
\text { Classroom Marks }=\sum_{i=1}^{n} w_{i} * m_{i} \quad(i=1,2 . . n)
$$

In (1),

$$
\sum_{i=1}^{n} w_{i}=1
$$

Thirdly, according to the marks of each available, the top 10 classrooms are chosen as recommended classroom and they will be sent to the user. Further more, when user chooses a classroom, the system will provide a shortest distance way for the student to get to the destination.

At last, after a user receives the results and chooses his favorite one, his choice will send back to the server. According to user's choice, the system will update the weight value of each factor and saved them as the personal profile in the database. Consequently, each user of this system will have his personal profile and have different experience.

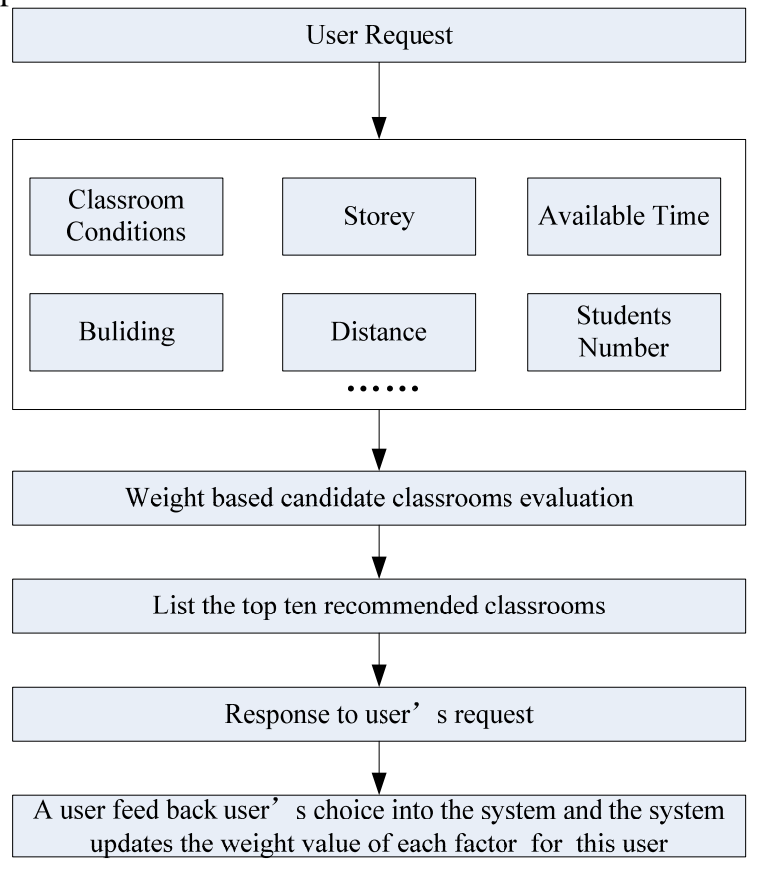

Figure 3. Weight based available classroom searching method

\section{IMPLEMENTATION OF CACS}

Based on the system model that we have discussed in the previous sections, we construct a prototype system of CACS. In CACS, the available classrooms are listed in a descending order according to the evaluated marks. But the user may not accept the system recommended best classroom with the highest marks and choose his favorite one among the other candidate classrooms. After completing the operation of choosing a classroom, the user's action will be fed back to the system and then the user's personal preference records will be updated.

We also provide a textbox for users to type in his current locations, because the user's device may not have a GPS module. Further more, we provide an interface for users to set which factors should be take into consideration. 
The users regard that this system provides them an efficiency way to find an available classroom.

\section{CONCLUSION}

In this paper, we design and implementation of a contextaware available classrooms searching system. Different with previous system, this system can provide users the recommended classrooms according to users' preferences, and users can enjoy a good experience. In the further work we will introduce machine learning algorithms to make the weight of each factor more accurate and take more factors of users preferences into consideration to make the system more efficiently.

\section{ACKNOWLEDGMENT}

This work is supported by "the Fundamental Research Funds for the Central Universities” under Grant No. 529020900201089, and by "the Fundamental Research Funds for the Central Universities” under Grant No.52204-10161, and by "the National Undergraduate Training Programs for Innovation and Entrepreneurship" under Grant No. 201210504022.

\section{REFERENCES}

[1] J. E. Bardram, "Applications of context-aware computing in hospital work - Examples and design principles," Proceedings of the ACM Symposium on Applied Computing. 2004, pp. 1574-1579.

[2] P. M. Barnaghi, and S. A. Kareem, "A context-aware ranking method for the complex relationships on the semantic web," Proceedings of the 6th International Conference on Advanced Language Processing and Web Information Technology, 2007, pp. 129-134.

[3] P.-C. Huang, and Y.-H. Kuo, "A reliable context model for contextaware applications," IEEE International Conference on Systems, Man and Cybernetics, 2008, pp. 246-250.

[4] R. Lowe, P. Mandl, and M. Weber, "Context Directory: A contextaware service for mobile context-aware computing applications by the example of Google Android," 2012 IEEE International Conference on Pervasive Computing and Communications Workshops, 2012, pp. 76-81.

[5] X. Hua, Z. Ying, J. Ng et al., "An Approach for Context-Aware Service Discovery and Recommendation," 2010 IEEE International Conference on Web Services (ICWS), 2010, pp. 163-170.

[6] P. V. Pushpa, and P. Venkataram, "Context aware M-commerce services: C-IOB model approach," The 8th International Conference on Information, Communications and Signal Processing (ICICS), 2011, pp. 1-5.

[7] G. S. Thyagaraju, and U. P. Kulkarni, "Modeling user context for Interactive Context Aware TV," IEEE International Conference on Computational Intelligence and Computing Research (ICCIC), 2010, pp. 1-5.

[8] L. Yonnim, and K. Ohbyung, "Criteria for Measuring Information Privacy in Context-Aware Computing Environments," The third International Conference on Multimedia and Ubiquitous Engineering, 2009, pp. 158-163.

[9] G. Pallapa, M. Di Francesco, and S. K. Das, "Adaptive and contextaware privacy preservation schemes exploiting user interactions in pervasive environments," IEEE International Symposium on a World of Wireless, Mobile and Multimedia Networks (WoWMoM), 2012, pp. 1-6. 